\title{
Endocytose sans clathrine
}

\section{La voie est libre !}

Cédric M. Blouin

> L'intérêt porté à l'étude des mécanismes d'endocytose n'a jamais faibli tant ce phénomène tient une place essentielle dans le fonctionnement cellulaire. La croissance, l'adhésion et la différenciation cellulaires, la régulation de la signalisation induite par les récepteurs membranaires ou encore l'infection par des particules virales, dépendent toutes de l'entrée de molécules dans la cellule. Une fois l'endocytose dépendante de la clathrine bien caractérisée, il est apparu que d'autres processus d'entrée, qui ne mettent pas en jeu la clathrine, existent également dans la cellule. Cette synthèse a pour but de faire le point sur les avancées récentes qui établissent avec certitude l'existence des voies d'endocytose indépendantes de la clathrine, et qui mettent en évidence leurs régulations spécifiques. <

Dans la cellule, il existe plusieurs voies permettant l'entrée de molécules provenant du milieu extérieur. En particulier, l'endocytose [1] $(\rightarrow)$ conduit à l'internalisation de protéines membranaires, de $(\rightarrow)$ Voir $m / \mathrm{s} \mathrm{n}^{\circ} 11$, vol. 18, novembre 2002, page 1126 récepteurs et de leurs ligands. Jusqu'à la moitié des années 1990, l'existence de voies d'endocytose distinctes de la voie classique qui fait intervenir la clathrine [2] $(\rightarrow)$ restait controversée. Dans un premier temps, l'utilisation d'agents pharmacologiques perturbant la voie

$\rightarrow$ Voir $m / s n^{\circ} 2$ vol. 27, février 2011, page 122

d'endocytose classique a conduit à formuler l'hypothèse de l'existence d'une voie alternative. Par la suite, l'utilisation d'outils génétiques ciblant sélectivement les acteurs de la formation des puits recouverts de clathrine confirma définitivement leur existence [3]. Dès 2001 , les premières protéines « cargo » empruntant des voies d'endocytose indépendantes de la clathrine furent identifiées [4-6]. Désormais, ces voies multiples sont reconnues et commencent à être bien caractérisées. On sait maintenant qu'elles existent dans l'en-

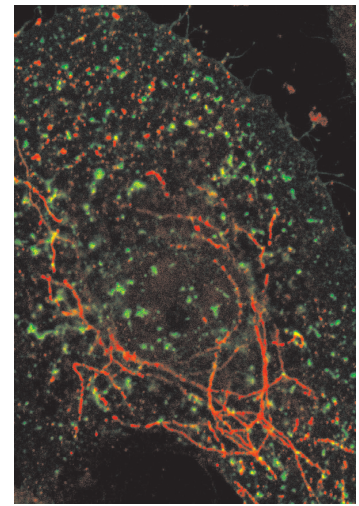

Institut Curie, CNRS UMR144, Laboratoire Trafic, signalisation et ciblages intracellulaires, 26, rue d'Ulm, 75248 Paris Cedex 05, France. cedric.blouin@curie.fr

semble des cellules eucaryotes, mais cette synthèse s'intéressera uniquement aux cellules de mammifères dans lesquelles ces voies sont les mieux décrites. Les voies d'endocytose indépendantes de la clathrine possèdent leurs régulateurs propres, mais elles partagent néanmoins des caractéristiques communes comme leur association à des microdomaines lipidiques membranaires, le rôle du cytosquelette d'actine et l'action régulatrice de petites GTPases (Tableau I, Figure 1).

\section{Endocytose indépendante de la clathrine et dépendante de la dynamine}

\section{Les cavéoles}

Les premières structures soupçonnées de participer aux phénomènes d'endocytose indépendants de la clathrine furent les cavéoles. Ces invaginations de la membrane plasmique de 60-80 nm présentent, en microscopie électronique, un aspect proche de celui des puits recouverts de clathrine [7]. De plus, elles partagent avec eux le recrutement de la dynamine impliquée dans le mécanisme de fission des vésicules lors de l'endocytose [8, 9]. Le constituant majeur des cavéoles est la protéine cavéoline-1 (CAVI) [10] $(\rightarrow)$. Deux autres membres complètent cette famille, CAV2 et CAV3, cette dernière étant

$(\rightarrow)$ Voir $m / s n^{\circ} 11$, vol. 27, novembre 2011, page 940 exprimée uniquement dans les cellules musculaires. La perte de CAVl et CAV3 conduit à la disparition des cavéoles, ce qui n'est pas le cas pour CAV2 [11]. Les cavéolines adoptent une conformation en épingle à cheveux dont les extrémités amino- et carboxy-terminales sont cytosoliques (Figure 2). Elles possèdent également plusieurs domaines qui permettent l'oligomérisation des cavéolines entre elles, et leur insertion dans la membrane plasmique [11]. Le caveolin scaffolding domain (CSD) permet des interactions avec de nombreuses molécules impliquées dans différentes voies de 


\begin{tabular}{|c|c|c|c|c|}
\hline Mécanisme & Voie d'endocytose & Morphologie & $\begin{array}{l}\text { Protéines } \\
\text { impliquées }\end{array}$ & Protéines 《 cargo » \\
\hline \multirow{2}{*}{$\begin{array}{l}\text { Dépendant de } \\
\text { la dynamine }\end{array}$} & Cavéoles & Vésiculaire & $\begin{array}{l}\text { Cavéoline-1,-2,-3 } \\
\text { Cavine-1,-2,-3,-4 } \\
\text { Src, PKC, actine }\end{array}$ & $\begin{array}{c}\text { Cholestérol, } \\
\text { glycosphingolipides, AMF, } \\
\text { lactosylcéramide, CTxB, SV40, } \\
\text { albumine }\end{array}$ \\
\hline & IL2-Rß & Vésiculaire? & $\begin{array}{l}\text { RhoA, RhoGDI, Racl, } \\
\text { PAK1, PAK2, cortactine, } \\
\text { N-WASP, actine }\end{array}$ & $\begin{array}{c}\text { IL2-R } \beta \text {, } \\
\text { chaîne } \gamma \mathrm{c}, \\
\text { toxines Clostridium }\end{array}$ \\
\hline \multirow{4}{*}{$\begin{array}{l}\text { Indépendant } \\
\text { de la dynamine }\end{array}$} & CLIC-GદદC & $\begin{array}{l}\text { Tubulaire, } \\
\text { croissant }\end{array}$ & $\begin{array}{l}\text { Cdc42, Arfl, GRAFl, } \\
\text { actine }\end{array}$ & $\begin{array}{l}\text { Protéines à ancre GPI, } \\
\text { marqueurs de phase fluide }\end{array}$ \\
\hline & Arf6 & Vésiculaire? & Arf6, actine & $\begin{array}{c}\text { CD59, CMH-I, } \\
\text { carboxypeptidase } \varepsilon \text {, } \\
\text { intégrine } \beta 1, \\
\text { cadhérine } \varepsilon\end{array}$ \\
\hline & Flotillines & Vésiculaire & $\begin{array}{l}\text { Flotilline-1,-2 } \\
\text { Actine }\end{array}$ & Protéines à ancre GPI, CTxB \\
\hline & $\begin{array}{l}\text { Toxine } \\
\text { de Shiga }\end{array}$ & Tubulaire & Actine & $\mathrm{ST} \times \mathrm{B}, \mathrm{CT} \times \mathrm{B}, \mathrm{SV} 40$ \\
\hline
\end{tabular}

Tableau I. Caractéristiques moléculaires et morphologiques des différentes voies d'endocytose indépendantes de la clathrine.

signalisation, bien que cette propriété soit néanmoins controversée par des travaux récents [12]. La formation de cavéoles semble initiée au niveau de l'appareil de Golgi et nécessite l'oligomérisation de cavéolines en association avec des microdomaines riches en cholestérol (que CAVl peut lier directement), en glycosphingolipides (gangliosides GMl et GM3) et en sphingolipides [13]. En résumé, les cavéolines stabilisent des domaines lipidiques qui constituent une phase liquide ordonnée non miscible avec le reste des lipides membranaires appartenant à la phase fluide.

Récemment, une nouvelle famille de protéines cytosoliques nécessaires à l'assemblage des cavéoles a été identifiée : les cavines. Elle comprend la cavine-l ou polymerase I and transcript release factor (PTRF), la cavine-2 ou serum deprivation protein response (SDPR), la cavine-3 ou sdr-related gene product that binds to C-kinase (SRBC) et la cavine-4 ou muscle restricted coiled-coil protein (MURC) [14]. Les cavines interagissent directement avec les cavéolines pour maintenir la structure des cavéoles. En leur absence, les cavéoles se désassemblent, conduisant à l'endocytose des cavéolines pour leur dégradation. Plus précisément, la cavine-1 jouerait un rôle dans la formation des cavéoles, la cavine-2 dans l'initiation de leur courbure et la cavine-3 dans la mise en place de l'endocytose cavéolaire [15]. Depuis l'hypothèse initiale de son existence, l'endocytose par les cavéoles est sujette à controverse en raison notamment de l'absence de protéine «cargo » identifiée utilisant spécifiquement cette voie [16]. En 2001, des travaux ont montré l'endocytose du virus simien 40 (SV40) via les cavéoles vers un compartiment endosomal: le cavéosome [17]. Pour autant, des éléments récents remettent en cause la spécificité cavéolaire de l'endocytose du SV40. En effet, la même équipe a pu montrer ensuite que l'entrée du SV40 dans des cellules dépourvues de cavéoline-1 (lignée cellulaire hépatique humaine $\mathrm{HuH7}$ et fibroblastes embryonnaires de souris $\mathrm{Cavl}^{-/-}$) se faisait par une voie alternative, dépendante du cholestérol et de protéines à activité tyrosine kinase, mais indépendante de la clathrine, de la dynamine et de Arf6 (ADP-ribosylation factor 6) [18]. Par ailleurs, ces auteurs ont montré que le cavéosome n'était pas un compartiment nouveau, mais correspondait en fait à l'accumulation de cavéolines dans les corps multivésiculaires avant qu'elles soient dégradées. Ce phénomène est observable en réponse à la surexpression de CAVl, à la déstabilisation des cavéoles par la perturbation du cholestérol ou à la diminution de la quantité de cavines disponibles [19]. Enfin, on sait désormais que le ganglioside GMl, récepteur lipidique enrichi dans les cavéoles, mais présent également dans d'autres microdomaines lipidiques et dans les puits à clathrine [11], est suffisant pour l'entrée de SV40 dans la cellule. La liaison d'un pentamère de la protéine VPl, formant la capside du virus, aux GMl à chaînes acylées longues induit la formation d'invaginations membranaires, puis l'endocytose de la particule virale par une voie d'endocytose différente que nous décrirons plus loin [20]. 


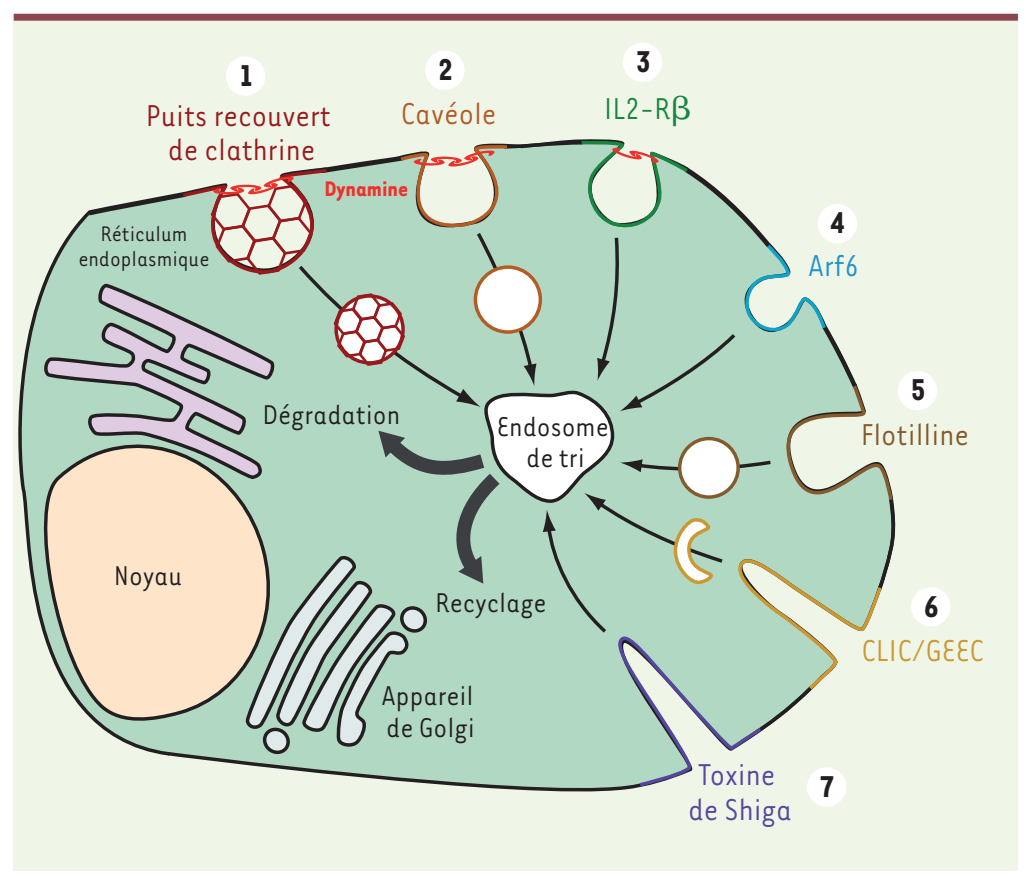

Figure 1. Voies d'endocytose et trafic intracellulaire. $\varepsilon$ n parallèle des puits recouverts de clathrine (1), plusieurs autres voies d'endocytose existent. Parmi elles, certaines nécessitent l'intervention de la dynamine pour séparer les vésicules de la membrane plasmique. C'est le cas de l'endocytose par les cavéoles (2), invaginations recouvertes de cavéolines et enrichies en cholestérol et en sphingolipides, ainsi que de la voie d'endocytose du récepteur IL2-R $\beta$ (3). D'autres voies d'entrée ne font pas intervenir la dynamine, mais nécessitent la présence de microdomaines lipidiques. Elles présentent également leurs spécificités : l'activité de Arf6 (4), la présence d'un manteau de flotillines (5), l'endocytose de protéines à ancre GPI via des intermédiaires de transport en forme de croissant (6) ou des invaginations propres à certaines toxines (7). Une fois atteint l'endosome de tri, les protéines « cargo » internalisées par ces voies d'endocytose multiples sont adressées vers la dégradation lysosomale ou le recyclage vers la membrane plasmique.

Si les cavéoles sont des structures beaucoup plus stables que les puits de clathrine, il apparaît que la dynamine participe à leur internalisation sous condition, notamment, de stimulation par l'ajout de stérols et de glycosphingolipides [21]. D'autres facteurs sont nécessaires à l'endocytose des cavéoles: la kinase Src, la protéine kinase C $\alpha$ (PKC $\alpha$ ) et le recrutement d'actine [11]. L'endocytose cavéolaire peut être régulée par le niveau d'expression de CAVI. En cas de déplétion, l'endocytose est inhibée en raison de la perturbation du contenu en cholestérol des cellules et de la désorganisation des domaines lipidiques à la membrane plasmique. Le même effet est observé lors d'une surexpression de CAVl $[22,23]$. Plusieurs protéines « cargo » empruntant une voie cavéolaire, sans que celle-ci soit exclusive, ont été identifiées. C'est le cas du facteur autocrine de mobilité (AMF) qui, une fois internalisé, parvient au réticulum endoplasmique, ainsi que du lactosylcéramide $[23,24]$. Ces mêmes travaux ont montré que l'entrée de la sous-unité $B$ de la toxine cholérique (CTXB) dépendait du niveau d'expression de CAVI. Si ce dernier est faible, CTxB est endocytosée via une voie dépendante de la clathrine pour atteindre l'appareil de Golgi. Les cavéoles sont également impliquées dans le mécanisme de transcytose qui intervient dans les cellules endothéliales. Ce processus permet le transport de protéines « cargo » d'un milieu extracellulaire vers un autre à travers une cellule polarisée. Bien que le terme cavéole ne soit pas employé, de l'albumine marquée à l'or perfusée dans les vaisseaux fut observée par microscopie électronique au sein de vésicules caractéristiques dans les capillaires sanguins [25]. Récemment, cette voie a été démontrée grâce à la visualisation in vivo de la transcytose de l'aminopeptidase $P$ (APP) de la lumière du vaisseau sanguin vers le tissu pulmonaire environnant à travers I'endothélium [26].

Des travaux récents ont mis à jour un rôle nouveau des cavéoles qui devrait conduire à reconsidérer leurs fonctions cellulaires classiques.
En effet, lors d'un stress mécanique de la membrane plasmique induit par étirement ou par choc osmotique, les cavéoles s'aplanissent immédiatement dans la membrane, ce qui rend disponible la quantité de membrane additionnelle stockée dans l'invagination cavéolaire, et évite ainsi l'augmentation de la tension de membrane préjudiciable à la cellule. Les cavéoles sont ainsi des mécanosenseurs nécessaires au maintien de l'homéostasie de la tension membranaire durant le stress mécanique [27]. Cette fonction mécanique nouvelle des cavéoles est en accord avec son faible niveau d'endocytose à l'état basal qui permet la formation d'un réservoir membranaire stable à la membrane plasmique. Il reste maintenant à comprendre comment intégrer le rôle mécanique des cavéoles avec leurs fonctions classiques dans les domaines de la signalisation et du trafic membranaire [28].

\section{Endocytose du récepteur de l'interleukine-2}

La première voie d'endocytose indépendante de la clathrine et de la cavéoline identifiée est celle de la chaîne $\beta$ du récepteur de l'interleukine 2 (IL2-R $\beta$ ) [4]. Après liaison de son ligand, I'IL2-R $\beta$ s'associe à la fraction membranaire résistante aux détergents (DRM), enrichie en cholestérol et en certains sphingolipides. Cette voie est donc sensible au cholestérol, mais nécessite également l'activité de la dynamine et des petites protéines $G$ RhoA et Racl [4, 29]. D'autres régulateurs de l'endocytose de l'IL2-R $\beta$ sont désormais connus : les p21-activated kinases PAK1 et PAK2, qui interviennent dans le remodelage du cytosquelette d'actine via la 


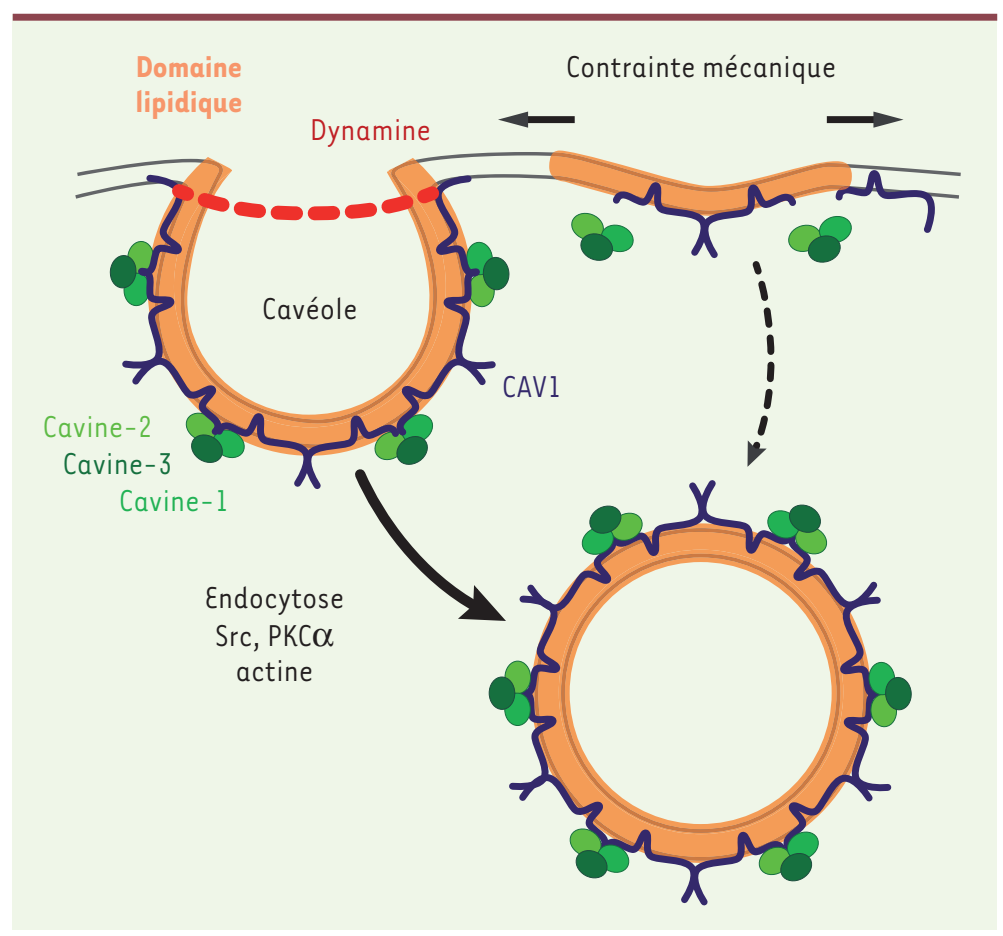

Figure 2. Cavéolines et cavéoles: structure, endocytose et mécanosensibilité. Les oligomères de cavéoline-1 (CAV1), en association avec des microdomaines lipidiques riches en cholestérol et en sphingolipides, forment les cavéoles, invaginations à la membrane plasmique de 60-80 nm. Les cavines-1, -2 et -3 interagiraient avec CAVI pour donner aux cavéoles leur forme et leur courbure, et permettre leur endocytose. Cette dernière peut être induite par certaines protéines « cargo » comme les particules virales et l'ajout de lipides; elle dépend de l'action de la dynamine, des kinases Src et PKC $\alpha$, ainsi que de l'actine. Sous l'effet d'une contrainte mécanique, les cavéoles s'aplanissent par un processus passif ne nécessitant pas l'utilisation d'ATP. Elles assurent ainsi l'homéostasie de la tension membranaire de la cellule. Ceci a pour conséquence la libération de CAVl libre et des cavines cytosoliques. Le lien entre l'endocytose cavéolaire et le rôle de mécanosenseur des cavéoles reste à déterminer, même si on peut penser que le maintien de l'endocytose à un niveau faible permet à la cellule de conserver une plus grande réserve potentielle de membrane.

phosphorylation de la cortactine, et la protéine du syndrome neuronal Wiskott-Aldrich (N-WASP) [29, 30]. Cette voie est certainement partagée avec d'autres récepteurs de cytokines, notamment la chaîne $\gamma c$, qui est commune à plusieurs récepteurs de cytokines [31]. Certaines toxines bactériennes du genre Clostridium utilisent cette voie pour intoxiquer les cellules [32].

\section{Endocytose indépendante de la dynamine}

L'inhibition de la dynamine, par l'utilisation de mutants dominants négatifs, ne bloque pas entièrement l'entrée de molécules solubles (phase fluide) dans la cellule [33]. Ceci suggère l'existence de voies d'endocytose dont le processus de détachement vésiculaire de la membrane plasmique ne nécessite pas l'intervention de cette GTPase.

\section{La voie CLIC/GEعC}

Certaines protéines s'insèrent dans le feuillet externe de la membrane plasmique grâce à une ancre lipidique de type glycosylphosphatidylinositol (GPI). Elles sont présentes sous forme de monomères, mais également sous forme de clusters de quelques nanomètres associés à des microdomaines lipidiques enrichis en cholestérol et en sphingolipides [34]. L'internalisation des protéines à ancre GPI (GPI-AP) n'est pas perturbée lorsque la formation des puits à clathrine est inhibée, définissant ainsi une nouvelle voie indépendante de la clathrine (clathrin-independent carriers, CLIC). L'internalisation des CLIC conduit à la création d'un compartiment endosomal précoce enrichi en GPI-AP, le GEદC (GPI-AP enriched endocytic compartment), distinct de l'endosome précoce car dépourvu du marqueur Rab5. Les intermédiaires d'endocytose de la voie CLIC contenant les GPI-AP présentent une forme tubulaire caractéristique en anneau ou croissant. La voie d'endocytose CLIC se différencie de celle de I'IL2-R car elle est indépendante de la dynamine et de RhoA, mais régulée par les petites GTPases Cdc42 et Arfl [3537] $(\rightarrow)$. Par la suite, GRAFl (GTPase regulator associated $\rightarrow$ ) Voir $m / s n^{\circ} 8-9$, vol. 21 août-septembre 2005 , page 692 with focal adhesion kinase 1), une protéine à domaine BAR (Bin-Amphiphysin-Rvs), fut identifiée sur les structures tubulaires contenant des GPI-AP internalisées et associées à l'activité de $\mathrm{Cdc42}$. Cette découverte permet désormais de mieux comprendre comment sont formés et stabilisés les tubules de la voie CLIC, mais aussi de disposer d'un marqueur spécifique de cette voie [38].

\section{Les flotillines}

Les flotillines- 1 et -2 présentent une topologie similaire à celle des cavéolines et, comme elles, sont associées à des microdomaines lipidiques riches en cholestérol et en sphingolipides. Néanmoins, ces protéines membranaires délimitent des domaines distincts des cavéoles. La déplétion de la flotilline-l endogène inhibe partiellement l'entrée de CTXB et l'endocytose des GPI-AP dans les fibroblastes de souris [39]. La voie d'endocytose dépendante des flotillines apparaît comme indépendante de la dynamine, car l'inhibition de l'endocytose de CTXB est d'autant plus forte lors de la surexpression d'un dominant négatif de la dynamine (DynK44A) [39]. L'assemblage des flotillines est également capable d'induire la formation d'invaginations morphologiquement 


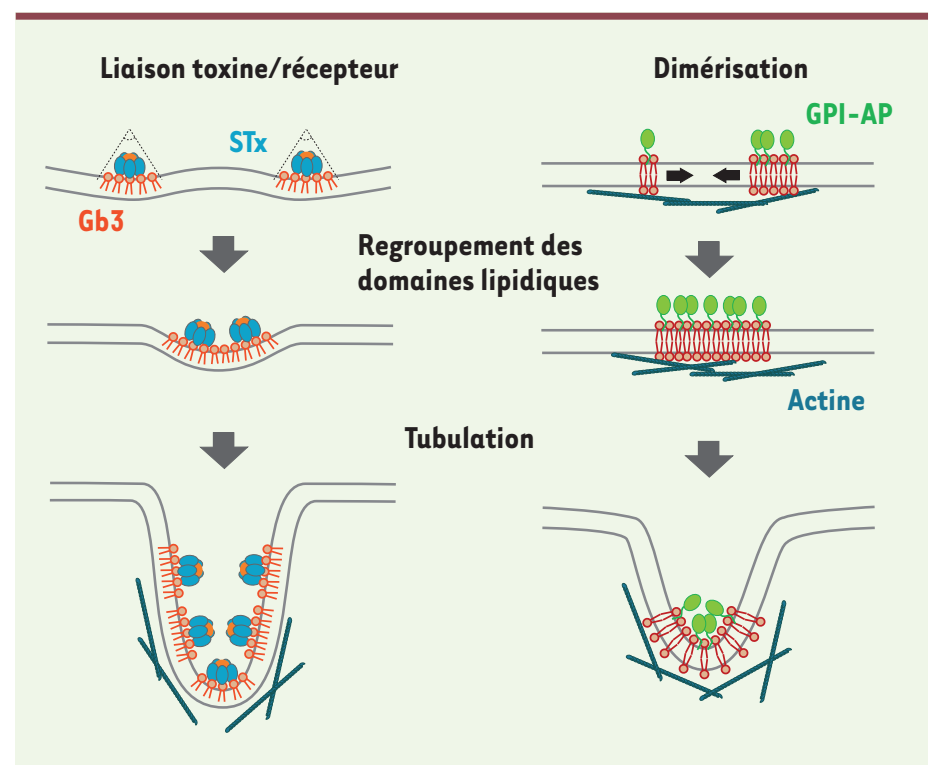

Figure 3. Concentration et endocytose des protéines « cargo 》 associées aux microdomaines lipidiques. La liaison de la toxine de Shiga (STx) à son récepteur lipidique Gb3 provoque la compression du feuillet externe de la membrane plasmique, ce qui crée des perturbations locales. Pour réduire l'énergie de tension de ligne aux frontières des domaines lipidiques hétérogènes, les complexes toxines/récepteurs se rassemblent pour former des domaines plus importants. Pour les protéines à ancre GPI (GPI-AP), la concentration des protéines «cargo » se produit grâce à un phénomène de dimérisation stabilisé par le cytosquelette d'actine et les microdomaines lipidiques. Dans les deux cas, c'est l'invagination des microdomaines lipidiques qui permettra de réduire l'énergie de tension de ligne et provoquera, après scission, l'entrée des molécules dans la cellule.

proches des cavéoles et l'accumulation de vésicules intracellulaires participant aux processus d'endocytose indépendants de la dynamine [40]. À l'instar des cavéolines, les flotillines sont vraisemblablement à l'origine de domaines lipidiques comme le montre la localisation de ces protéines dans les membranes d'intermédiaires précoces de la voie CLIC/GEEC [38]. Cependant, l'absence de protéine « cargo » spécifique de ces domaines laisse un doute sur leur capacité réelle d'endocytose.

\section{ADP-ribosylation factor 6 (Arf6)}

En 1997, la surexpression de mutants d'Arf6 à la membrane plasmique a permis de mettre en évidence une voie d'entrée utilisée par des protéines membranaires dépourvues de motifs susceptibles d'interagir avec le complexe AP2 (adaptor protein complex 2)/ clathrine [41]. C'est le cas de CD59, une autre protéine endogène à ancre GPI, et du complexe majeur d'histocompatibilité de classe-I $(\mathrm{CMH}-\mathrm{I})$. Après endocytose, ils sont rapidement localisés dans des endosomes enrichis en Arf6 et en cholestérol avant de rejoindre, dans les endosomes de tri, le récepteur à la transferrine, une protéine « cargo » modèle de la voie clathrine [42]. Bien que CD59 soit une GPI-AP, ces structures endosomales sont distinctes de celles de la voie CLIC/G\&EC décrite précédemment [42]. D'autres protéines
« cargo » ont été identifiées dans la voie dépendante d'Arf6: la carboxypeptidase $\varepsilon$, l'intégrine $\beta 1$ et la cadhérine $\varepsilon$ [43-45]. Néanmoins, il apparaît aussi que, dans cette voie liée à Arf6, ce ne soit pas le processus d'endocytose qui dépend de l'activation d'Arf6 (Arf6-GTP) ou de son inactivation, mais plutôt le recyclage vers la membrane plasmique des protéines «cargo » qui l'empruntent. En effet, l'emploi de la forme mutée non hydrolysable $067 \mathrm{~L}$ induit l'accumulation des protéines « cargo », $\mathrm{CMH}-\mathrm{l}$ et intégrine $\beta 1$, dans des vacuoles recouvertes d'actine et enrichies en phosphatidylinositol 4,5-bisphosphate (PIP2) [44].

\section{Les microdomaines lipidiques à l'origine de l'invagination et de la scission}

Pour ces voies d'endocytose qui n'impliquent pas de protéines de manteau classiques, comme la clathrine ou la cavéoline, deux questions demeurent : par quels mécanismes les protéines « cargo » sont-elles concentrées avant leur endocytose? Comment la membrane plasmique est-elle déformée pour induire la tubulation puis la scission nécessaires à l'endocytose des protéines «cargo »? Des études récentes révèlent le rôle primordial des microdomaines lipidiques qui sont présents dans toutes les voies d'endocytose indépendantes de la clathrine. Afin de minimiser l'énergie résultant des perturbations locales de la membrane plasmique (disparité de composition ou d'épaisseur, déformation), ces domaines auront tendance à se rassembler. Les protéines «cargo » qui leur sont associées sont ainsi rassemblées au sein de domaines de taille plus importante [46]. Ce cas de figure est parfaitement illustré par la liaison de la toxine de Shiga (STx, produite par Shigella dysenteriae) au glycosphingolipide Gb3, son récepteur spécifique [47]. Ainsi, la liaison de la sous-unité B de la STx, qui adopte une structure pentamérique caractéristique, induit la compression du feuillet externe de la membrane plasmique. Ceci entraîne la création d'asymétries locales qui se traduisent par une courbure négative importante de la membrane plasmique vers l'intérieur de la cellule (Figure 3). L'accumulation de protéines « cargo » peut également se faire selon un processus actif, comme pour les GPI-AP qui sont présentes au niveau des microdomaines lipidiques sous forme de clusters ou de monomères, et qui peuvent être rassemblés sous l'action du cytosquelette d'actine corticale [48]. II a également été proposé que le cholestérol permette la stabilisation d'homodimères qui ne sont formés que de manière transitoire en son absence [49]. 
L'invagination des microdomaines lipidiques permet également de réduire l'énergie d'interface à leurs extrémités dû au phénomène de «tension de ligne» [50]. D’autres mécanismes peuvent entrer en jeu pour déformer la membrane, comme la transformation de lipides en d'autres espèces de conformation différente. II est aussi possible d'observer, dans certains cas, que la courbure de la membrane induit elle-même une partition des espèces lipidiques [46].

La dynamine joue un rôle essentiel dans le processus de scission des invaginations formées au cours de l'endocytose dépendante de la clathrine ou de la cavéoline, et l'endocytose du récepteur IL2-R $\beta$. Néanmoins, la dynamine n'intervient pas dans les autres voies décrites dans cette revue. Initiés chez la levure, qui ne possède pas de dynamine, de nombreux travaux ont cherché à comprendre comment des modifications de la membrane plasmique pouvaient aboutir à la formation de vésicules. Il apparaît que la tension de ligne est, là encore, un élément fondamental pour expliquer la scission des vésicules [51]. Cela a été démontré d'abord in vitro grâce à un système de membranes modèles, puis in vivo dans le contexte de l'endocytose de la STx $[52,53]$. La polymérisation de l'actine participe à ce phénomène en réorganisant la membrane et en formant des domaines lipidiques distincts, à la frontière desquels pourrait avoir lieu la scission [53].

\section{Conclusion}

Longtemps sous-estimées voire ignorées, les voies d'endocytose indépendantes de la clathrine voient aujourd'hui leur existence reconnue. Elles conservent cependant encore une grande part de mystère et beaucoup de questions essentielles demeurent. Un schéma commun apparaît néanmoins qui fait intervenir plusieurs processus intimement liés. On notera, tout d'abord, la concentration des protéines « cargo » induite par leur liaison aux récepteurs/adapteurs protéiques ou lipidiques. Leur association avec des microdomaines lipidiques riches en glycosphingolipides et en cholestérol permet leur partition et l'initiation de la courbure de la membrane plasmique et, dans certains cas, la scission des vésicules. II faut également compter sur l'action des petites protéines GTPases qui régulent la polymérisation de l'actine et les modifications du cytosquelette. Des questions subsistent quant à la spécificité ou la flexibilité de chaque voie décrite dans cette synthèse, tant les molécules pouvant emprunter plusieurs chemins sont nombreuses. Les études futures auront aussi pour but de comprendre le rôle des autres voies dans les grandes fonctions cellulaires comme, par exemple, leur lien avec l'initiation de voies de signalisation intracellulaire [54]. $\diamond$

\section{SUMMARY}

Clathrin-independent endocytosis: free the way!

Along the years, the interest paid to the study of endocytosis has never wavered as this process plays such an essential role in many cellular functions. Cell growth, adhesion and differentiation, regulation of signaling induced by membrane receptors or infection by viral particles are all dependent on the entry of molecules into the cell. Once the clathrin-dependent endocytosis well characterized, it has become apparent that other entry pathways also existed in the cell. This review is intended to provide an update on recent advances that establish with certainty the existence of endocytic pathways independent of clathrin and highlight their specific regulators. $\diamond$

\section{LIENS D'INTÉRÊT}

L'auteur déclare n'avoir aucun lien d'intérêt avec les données publiées dans cet article.

\section{REMERCIEMENTS}

L'auteur souhaite remercier Christophe Lamaze ainsi que l'ensemble du laboratoire "Trafic, signalisation et ciblages intracellulaires ». Il est soutenu par une bourse de la Ligue nationale contre le cancer.

\section{RÉFÉRENCES}

1. Benmerah A, Lamaze C. Endocytose : chaque voie compte! Med Sci (Paris) $2002 ; 18: 1126-36$.

2. Boucrot $\varepsilon$, McMahon $H T$. Initiation de l'endocytose par vésicules de clathrine : des « sculpteurs de membrane » au travail. Med Sci (Paris) $2011 ; 27$ : 122-5.

3. Lamaze C, Schmid SL. The emergence of clathrin-independent pinocytic pathways. Curr Opin Cell Biol $1995 ; 7: 573-80$.

4. Lamaze C, Dujeancourt A, Baba T, et al. Interleukin 2 receptors and detergent-resistant membrane domains define a clathrin-independent endocytic pathway. Mol Cell $2001 ; 7: 661-71$.

5. Nichols BJ, Kenworthy AK, Polishchuk RS, et al. Rapid cycling of lipid raft markers between the cell surface and Golgi complex.J Cell Biol 2001 ; 153 : 529-41.

6. Torgersen ML, Skretting G, van Deurs B, Sandvig K. Internalization of cholera toxin by different endocytic mechanisms. J Cell Sci 2001 ; 114 : 3737-47.

7. Palade GE. The fine structure of blood capillaries. J Appl Physiol $1953 ; 24$ : 1424.

8. Henley JR, Krueger EW, Oswald BJ, McNiven MA. Dynamin-mediated internalization of caveolae. J Cell Biol 1998; 141 : 85-99.

9. Oh P, McIntosh DP, Schnitzer JE. Dynamin at the neck of caveolae mediates their budding to form transport vesicles by GTP-driven fission from the plasma membrane of endothelium. J Cell Biol $1998 ; 141: 101-14$.

10. Goetz JG, Del Pozo MA. La cavéoline-1 force le remodelage de la matrice extracellulaire. Med Sci (Paris) $2011 ; 27: 940-4$

11. Parton RG, Simons K. The multiple faces of caveolae. Nat Rev Mol Cell Biol $2007 ; 8$ : 185-94.

12. Collins BM, Davis MJ, Hancock JF, Parton RG. Structure-based reassessment of the caveolin signaling model: do caveolae regulate signaling through caveolin-protein interactions? Dev Cell $2012 ; 23: 11-20$.

13. Ortegren $U$, Karlsson M, Blazic N, et al. Lipids and glycosphingolipids in caveolae and surrounding plasma membrane of primary rat adipocytes. Eur J Biochem $2004 ; 271: 2028-36$.

14. Hansen CG, Nichols BJ. Exploring the caves: cavins, caveolins and caveolae. Trends Cell Biol $2010 ; 20: 177-86$.

15. Parton RG, Del Pozo MA. Caveolae as plasma membrane sensors, protectors and organizers. Nat Rev Mol Cell Biol $2013 ; 14$ : 98-112.

16. Echarri A, Del Pozo MA. Caveolae. Curr Biol 2012 ; 22 : R114-6.

17. Pelkmans $L$, Kartenbeck J, Helenius A. Caveolar endocytosis of simian virus 40 reveals a new two-step vesicular-transport pathway to the ER. Nat Cell Biol $2001 ; 3: 473-83$.

18. Damm EM, Pelkmans L, Kartenbeck J, et al. Clathrin- and caveolin-1independent endocytosis: entry of simian virus 40 into cells devoid of caveolae. J Cell Biol $2005 ; 168:$ 477-88.

19. Hayer A, Stoeber M, Ritz D, et al. Caveolin-1 is ubiquitinated and targeted to intralumenal vesicles in endolysosomes for degradation. J Cell Biol 2010 ; $191: 615-29$.

20. Ewers $H$, Romer W, Smith AE, et al. GMl structure determines SV40-induced membrane invagination and infection. Nat Cell Biol $2010 ; 12$ : 11-8. 


\section{RÉFÉRENCES}

21. Sharma DK, Brown JC, Choudhury A, et al. Selective stimulation of caveolar endocytosis by glycosphingolipids and cholesterol. Mol Biol Cell 2004 ; 15 : 3114-22

22. Le PU, Guay G, Altschuler Y, Nabi IR. Caveolin-1 is a negative regulator of caveolae-mediated endocytosis to the endoplasmic reticulum. J Biol Chem $2002 ; 277: 3371-9$.

23. Le PU, Nabi IR. Distinct caveolae-mediated endocytic pathways target the Golgi apparatus and the endoplasmic reticulum. J Cell Sci $2003 ; 116: 1059-71$.

24. Singh RD, Puri V, Valiyaveettil JT, et al. Selective caveolin-1-dependent endocytosis of glycosphingolipids. Mol Biol Cell $2003 ; 14: 3254-65$.

25. Ghitescu L, Fixman A, Simionescu M, Simionescu N. Specific binding sites for albumin restricted to plasmalemmal vesicles of continuous capillary endothelium: receptor-mediated transcytosis.J Cell Biol 1986; 102 : 1304-11.

26. Oh P, Borgstrom $P$, Witkiewicz $\mathrm{H}$, et al. Live dynamic imaging of caveolae pumping targeted antibody rapidly and specifically across endothelium in the lung. Nat Biotechnol $2007 ; 25: 327-37$.

27. Sinha B, Koster $D$, Ruez $R$, et al. Cells respond to mechanical stress by rapid disassembly of caveolae. Cell $2011 ; 144: 402-13$.

28. Nassoy P, Lamaze C. Stressing caveolae new role in cell mechanics. Trends Cell Biol $2012 ; 22$ : 381-9.

29. Grassart A, Dujeancourt A, Lazarow PB, et al. Clathrin-independent endocytosis used by the IL-2 receptor is regulated by Racl, Pakl and Pak2. EMBO Rep 2008; 9: 356-62.

30. Grassart A, Meas-Yedid V, Dufour A, et al. Pakl phosphorylation enhances cortactin-N-WASP interaction in clathrin-caveolin-independent endocytosis. Traffic $2010 ; 11$ : 1079-91.

31. Sauvonnet N, Dujeancourt A, Dautry-Varsat A. Cortactin and dynamin are required for the clathrin-independent endocytosis of gammac cytokine receptor. / Cell Biol $2005 ; 168: 155-63$.

32. Gibert M, Monier MN, Ruez R, et al. Endocytosis and toxicity of clostridial binary toxins depend on a clathrin-independent pathway regulated by Rho-GDI. Cell Microbiol 2011 ; 13 : 154-70.

33. Damke H, Baba T, van der Bliek AM, Schmid SL. Clathrin-independent pinocytosis is induced in cells overexpressing a temperature-sensitive mutant of dynamin. J Cell Biol 1995; 131:69-80.

34. Sharma P, Varma R, Sarasij RC, et al. Nanoscale organization of multiple GPI-anchored proteins in living cell membranes. Cell $2004 ; 116: 577-89$.

35. Dubois T, Chavrier P. Une nouvelle protéine RhoGAP impliquée dans la régulation du complexe Arp2/3 au niveau de l'appareil de Golgi : un relais entre les protéines G ARFl et Cdc42. Med Sci (Paris) $2005 ; 21: 692-4$.

36. Sabharanjak S, Sharma P, Parton RG, Mayor S. GPI-anchored proteins are delivered to recycling endosomes via a distinct cdc42-regulated, clathrin-independent pinocytic pathway. Dev Cell $2002 ; 2: 411-23$.

37. Kumari S, Mayor S. ARFl is directly involved in dynamin-independent endocytosis. Nat Cell Biol $2008 ; 10: 30-41$.

38. Lundmark R, Doherty GJ, Howes MT, et al. The GTPase-activating protein GRAFl regulates the CLIC/ GદદC endocytic pathway. Curr Biol $2008 ; 18: 1802-8$.

39. Glebov 00, Bright NA, Nichols BJ. Flotillin-1 defines a clathrin-independent endocytic pathway in mammalian cells. Nat Cell Biol $2006 ; 8: 46-54$.

40. Frick M, Bright NA, Riento $K$, et al. Coassembly of flotillins induces formation of membrane microdomains, membrane curvature, and vesicle budding. Curr Biol 2007 ; 17 : 1151-6.
41. Radhakrishna H, Donaldson JG. ADP-ribosylation factor 6 regulates a nove plasma membrane recycling pathway. J Cell Biol 1997 ; 139 : 49-61.

42. Naslavsky N, Weigert R, Donaldson JG. Characterization of a nonclathrin endocytic pathway: membrane cargo and lipid requirements. Mol Biol Cell $2004 ; 15: 3542-52$

43. Arnaoutova I, Jackson CL, Al-Awar OS, et al. Recycling of Raft-associated prohormone sorting receptor carboxypeptidase $\varepsilon$ requires interaction with ARF6. Mol Biol Cell $2003 ; 14: 4448-57$.

44. Brown FD, Rozelle AL, Yin HL, et al. Phosphatidylinositol 4,5-bisphosphate and Arf6-regulated membrane traffic. J Cell Biol 2001 ; 154 : 1007-17.

45. Palacios F, Price L, Schweitzer J, et al. An essential role for ARF6-regulated membrane traffic in adherens junction turnover and epithelial cell migration. EMBO / $2001 ; 20$ : 4973-86.

46. Johannes L, Mayor S. Induced domain formation in endocytic invagination, lipid sorting, and scission. Cell $2010 ; 142$ : 507-10.

47. Romer W, Berland L, Chambon V, et al. Shiga toxin induces tubular membrane invaginations for its uptake into cells. Nature $2007 ; 450: 670-5$.

48. Goswami D, Gowrishankar K, Bilgrami S, et al. Nanoclusters of GPI-anchored proteins are formed by cortical actin-driven activity. Cell $2008 ; 135: 1085-$ 97.

49. Suzuki KG, Kasai RS, Hirosawa KM, et al. Transient GPI-anchored protein homodimers are units for raft organization and function. Nat Chem Biol $2012 ; 8: 774-83$.

50. Sarasij RC, Mayor S, Rao M. Chirality-induced budding: a raft-mediated mechanism for endocytosis and morphology of caveolae? Biophys / 2007 ; 92 : 3140-58.

51. Liu J, Sun Y, Drubin DG, Oster GF. The mechanochemistry of endocytosis. PLoS Biol $2009 ; 7$ : el000204.

52. Roux $A$, Cuvelier D, Nassoy $P$, et al. Role of curvature and phase transition in lipid sorting and fission of membrane tubules. EMBO J 2005 ; 24 : 1537-45.

53. Romer W, Pontani LL, Sorre B, et al. Actin dynamics drive membrane reorganization and scission in clathrin-independent endocytosis. Cell 2010 ; $140: 540-53$.

54. Gonnord P, Blouin CM, Lamaze C. Membrane trafficking and signaling: two sides of the same coin. Semin Cell Dev Biol $2011 ; 23$ : 154-64.

\section{TIRÉS À PART}

C.M. Blouin

\section{Bon de commande}

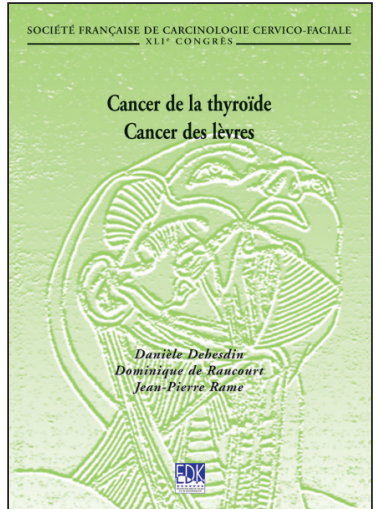

ISBN : 978-2-8425-4137-8 264 pages
À retourner à EDK, 25, rue Daviel - 75013 Paris

Tél. : 0158101905 - Fax : 0143293262 - E-mail : edk@edk.fr

NOM

Prénom :

Adresse :

Code postal :

Ville :

Pays :

Fonction :

Je souhaite recevoir l'ouvrage Cancer de la thyroïde - Cancers des lèvres : $35 €+3 €$ de port $=38 €$ TTC

en ................. exemplaire, soit un total de

$\square$ Par chèque, à l'ordre de $\mathbf{E} \mathbf{D} \mathbf{K}$

Par carte bancaire : $\quad \square$ Visa $\square$ Eurocard/Mastercard

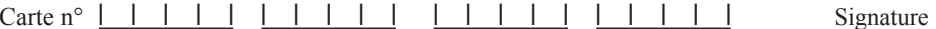

Date d'expiration: $\quad|\quad| 1|1|$

$N^{\circ}$ de contrôle au dos de la carte : $\quad|\quad| \quad \mid$ 